\title{
Research on Excessive Road Fuel Problem Based on Extension Strategy Generating Method
}

\author{
Li Shu fei, Ye Guang zai \\ Department of Computer Engineering, DongGuan polytechnic college, Dongguan, China
}

\begin{abstract}
The increase of the demand for gasoline in transportation and traffic vehicles results in excessive fuel consumption problem. However, by the extension strategy generating methods, the way of urban transportation changing from bus into metro may be analyzed and evaluated through extension reasoning, extension transformation and optimal calculation in order to solve the problem of excessive fuel consumption of the urban roads.
\end{abstract}

Index Terms: Fuel oil consumption; extension; strategy generating

(C) 2012 Published by MECS Publisher. Selection and/or peer review under responsibility of the Research Association of Modern Education and Computer Science

\section{Introduction}

With the development of the economic and extension of urban size, urban population and vehicle keep increasing [1]. Thus the urban transportation petrol oil consumption which adopts the vehicle as the main tools will increase accordingly. But the decreasing of the petrol oil day by day due to the non-renewability of petrol oil resources make people want to reduce the petrol oil consumption and prolong its service time. This research adopted extension strategy generating method [2], using formalized tools, through the combination of the qualitative \& quantitative to analyze the root cause and find out the effective and comparatively better strategy to solve the excessive urban transportation petrol oil consumption situation. And then find out the solution for the incompatible problem [2] of the increasing objective demands and the decreasing expectations.

\section{Extension Strategy Generating Method Basic Knowledge}

Extension strategy generating method is the process of generating strategy for incompatible problem based on the basic idea of Extension Theory to intimate human thinking model and use formalization \& quantification method [3].

* Corresponding author.

E-mail address: 1sfanny@tom.com, gddgygz@yahoo.com.cn 


\section{Extension strategy generating process}

The extension strategy generating theoretical principle is extension theory, the purpose of this theory is to solve those incompatible problem. The basic ideas are as follows:

(1) To define the target and factor of the existing problem, and set up problem extension strategy model with primitive representation.

(2) To determine the core problem according to the condition value (present value) and target value of the index provided by practical incompatible problem.

(3) To set up core problem compatible function and judge the incompatible level through calculation.

(4) If the core problem is incompatible, then making extension analyze on its target or condition to set up problem implication tree or interrelated tree[4] (Net).

(5) To make divergent or Conjugate analyze on implication tree or interrelated tree. Generate extension strategy generating tree through extension conversion and transmit conversion [5].

(6) To Calculate the problem tolerance degree after conversion, if tolerance degree change from less than 0 to no less than 0 , then incompatible problems convert into compatible problems. Namely the convert process is the strategy for the practical problem. is.

(7) To calculate the strategy goodness through related function. The bigger the value is the better the strategy

\section{Simple related function}

It's required to determine the tolerance degree or strategy goodness by quantitative calculation of the fixed problem during the extension strategy generating process. This research adopted simple related function [3] calculation method.

Positive field is finite interval $X=\langle a, b\rangle$, optimum value $x_{0} \in X$. Among the rest, when $x_{0}=a$, simple related function $k(x)$ at its maximum value, then

$$
k(x)= \begin{cases}\frac{x-a}{b-a}, & \mathrm{x}<\mathrm{a}, \\ \frac{b-x}{b-a}, & x>a, \\ k(a)=0 \vee 1, & x=a .\end{cases}
$$

This function can be used for solving problem degree of association (Function 1) .

\section{Goodness evaluation method} [6].

Goodness evaluation method is to evaluate a strategy capability through independent function quantification

Suppose the solution conformity to the problem I on measure condition $\mathrm{M}$ is $K(I)$, then

$$
k_{I}= \begin{cases}\frac{K(I)}{\max _{x \in X_{0}} K(x)}, & K(I)>0 \\ \frac{K(I)}{\max _{x \in X_{0}}|K(x)|}, & K(I)<0\end{cases}
$$

Become the standard conformity of solution I on M (Function 2). 
Suppose one problem measure condition is $M=\left\{M_{1}, M_{2}, \cdots, M_{n}\right\}$, Solution of I on M standard conformity is $k_{i}(i=1,2, \cdots n)$, Then $C(I)=\sum_{i=1}^{n} k_{i} \alpha_{i}$ is called problem solution I goodness.(Function 3).

\section{Research On Excessive Road Fuel Problem Based On Extension Strategy Generating Method}

To solve the problem of excessive road fuel problem, we combine extension strategy generating process to study strategy generating method.

\section{Core problem set up}

First we take the oil fuel consumption of city A as example. City A current oil fuel consumption is 20 million tons per year, it has reduce to 15 million tons per year according to energy saving requirement. Obviously there's a incompatible problem between the existing value (condition value) and target expecting value. In this case, we can use primitive to set up a core problem extenics model[2]: $p_{01}=g_{01} * l_{01}=$ (City A, target oil fuel consumption, 15 million tons/ year)* (City A, existing oil fuel consumption, 20 million tons/year). For city A's smallest oil fuel consumption is 0 million tons/year, and the optimal road oil fuel consumption is 0 million tons/year, then come to $a=0, b=15, x_{0}=0, x=20$, According to simple independent functions, as function 1 indicated, we can calculate the core problem association degree:

$$
k_{0}\left(p_{01}\right)=k(20)=\frac{15-20}{15-0}=-0.3333<0
$$

According to the above quantitative calculation of the core problem association degree, we get to know that the core problem association degree is less than 0 , so this problem is incompatible problem.

\section{Relative analysis}

If the core problem is incompatible, then we can make extension analyze on its target or condition to set up problem implication tree or correlation tree (net). This paper only does correlation analysis on the core problem condition and set up correlation tree.

As the urban oil fuel consumption is related to city centre branch numbers, vehicle type, automobile energy type, pavement condition index etc[1]. We can set up its correlation tree as Fig. 3-1 shows:

$$
P_{0}\left\{\begin{array}{l}
G_{0} \\
L_{0}\left\{\begin{array}{l}
R_{11} \\
R_{12} \\
R_{13} \\
R_{14}
\end{array}\right.
\end{array}\right.
$$

Fig. 3-1 Correlation Tree

Among them, $L_{0}=($ City A, Current road oil fuel consumption, V0) V0=<0, 17> Million tons/year

$\mathrm{R} 11=($ City A, city centre branches number, V01) V01 $=<1,10>$ Number

$\mathrm{R} 12=($ City A, main vehicle, V02) V02 $=\{$ Bus, Metro, car $\}$

$\mathrm{R} 13=($ City A, Vehicle energy type, V03 ) V03 $=\{$ Hybrid power[7], diesel oil, gasoline $\}$

R14=(City A, Vehicle energy type, V04) V04 $=$ sandy clay pavement, asphalt pavement, Cement pavement $\}$ 


\section{Strategy generating tree}

To make divergent analysis on correlation tree, we can get extension strategy generating tree by extension conversion and conduction conversion. So we can make divergent analysis on correlation tree and then make extension conversion and conduction conversion.

(1) Divergent analysis

Divergent analysis is divided into "One object more signs", "One signs more objects", "Same object same sign more value" etc[3]. many kinds of divergent analysis method. Here we use "same object same sign more value" method. Now we will make divergent analysis on related factors to conditional indicators. (i.e.:R11, R12, $\mathrm{R} 13, \mathrm{R} 14)$

$$
r_{111} \dashv\left\{\begin{array} { l } 
{ r _ { 1 1 2 } } \\
{ r _ { 1 1 3 } , r _ { 1 2 1 } \dashv }
\end{array} \left\{\begin{array} { l } 
{ r _ { 2 1 2 } } \\
{ r _ { 2 1 3 } , r _ { 1 3 1 } \dashv }
\end{array} \left\{\begin{array}{l}
r_{132} \\
r_{133}
\end{array}, r_{141} \leftrightharpoons \dashv\left\{\begin{array}{l}
r_{142} \\
r_{143}
\end{array}\right.\right.\right.\right.
$$

Among them, primitive $r_{111}$ is initial similar primitive of R11; primitive ${ }^{r_{121}}$ is initial similar primitive of R12; primitive $r_{131}$ is initial similar primitive of R13; primitive $r_{141}$ is initial similar primitive of R14;

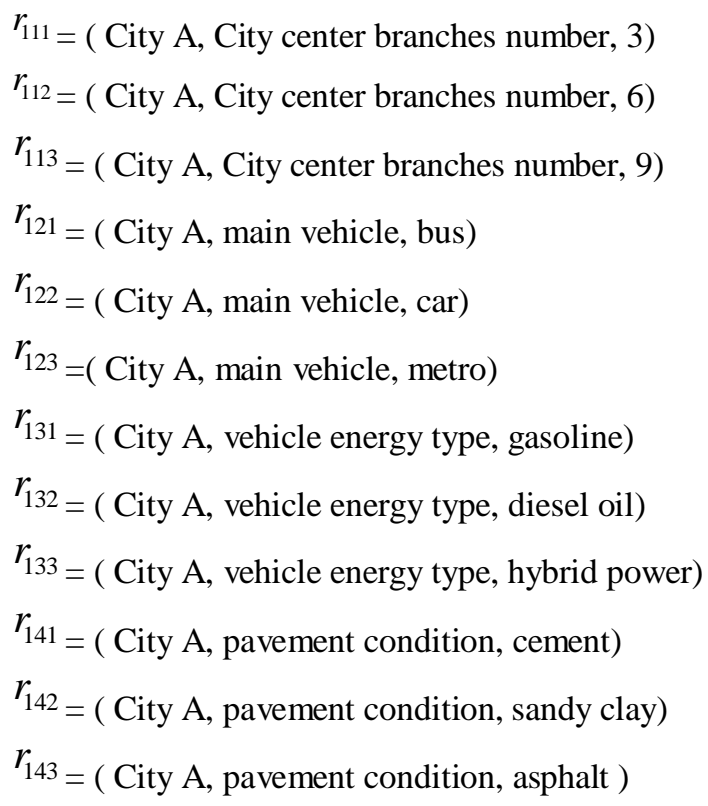

(2) Extension conversion and conduction conversion

After the divergent analysis, we can make extension conversion for initial primitives and then get new condition primitives through conduction conversion. This is the extension strategy generating process. The Fig. 3-2 is the corresponding extension strategy generating tree:

Fig. 3-2 Extension strategy generating tree

$$
{ }_{l_{0 i}} T_{p_{0 i}} \Leftarrow{ }_{r_{1 i 1}} T_{l_{0 i}} \Leftarrow \otimes\left[\begin{array}{l}
T_{r_{111}} \\
T_{r_{121}} \\
T_{r_{131}} \\
T_{r_{141}}
\end{array}\right.
$$

Extension conversion can indicate by affair-element, E.g. replace conversion $\varphi_{112}$ can indicated as: 


$$
\varphi_{112}=\left(\begin{array}{cc}
\text { replace, dominant object, } & r_{111} \\
\text { conversion result, } & r_{112}
\end{array}\right]
$$

It can also indicated with extension conversion as: $\varphi_{112} r_{111}=r_{112}$, due to the conduction effect, i.e. the conversion implication will result in the following conduction conversion: $\varphi_{112} \Rightarrow \varphi_{02}$, and $\varphi_{02} l_{01}=l_{02}$. We can get the following extension conversion and conversion implication:

$$
\begin{aligned}
& \varphi_{113} r_{111}=r_{113}, \varphi_{113} \Rightarrow \varphi_{03}, \varphi_{03} l_{01}=l_{03} ; \\
& \varphi_{122} r_{121}=r_{122}, \varphi_{122} \Rightarrow \varphi_{04}, \varphi_{04} l_{01}=l_{04} ; \\
& \varphi_{123} r_{121}=r_{123}, \varphi_{123} \Rightarrow \varphi_{05}, \varphi_{05} l_{01}=l_{05} ; \\
& \varphi_{132} r_{131}=r_{132}, \varphi_{132} \Rightarrow \varphi_{06}, \varphi_{06} l_{01}=l_{06} ; \\
& \varphi_{133} r_{131}=r_{133}, \varphi_{133} \Rightarrow \varphi_{07}, \varphi_{07} l_{01}=l_{07} ; \\
& \varphi_{142} r_{141}=r_{142}, \varphi_{142} \Rightarrow \varphi_{08}, \varphi_{08} l_{01}=l_{08} ; \\
& \varphi_{143} r_{141}=r_{143}, \varphi_{143} \Rightarrow \varphi_{09}, \varphi_{09} l_{01}=l_{09} .
\end{aligned}
$$

Among them: $l_{01}=$ ( City A, current road oil fuel consumption, 20 million tons/year);

$l_{02}=($ City A, current road oil fuel consumption, 16 million tons/year);

$l_{03}=($ City A, current road oil fuel consumption, 12 million tons/year);

$l_{04}=($ City A, current road oil fuel consumption, 80 million tons/year $) ;{ }^{05}=($ City A, current road oil fuel consumption, 11 million tons/year); ${ }^{l_{06}}=\left(\right.$ City A, current road oil fuel consumption, 17 million tons/year); ${ }^{07}=$ ( City A, current road oil fuel consumption, 13 million tons/year); $l_{08}=($ City A, current road oil fuel consumption,26 million tons/year); $l_{09}=$ ( City A, current road oil fuel consumption, 18 million tons/year);

(3) Problem association degree after conversion

The conversion of $\varphi_{112}$ result in the final city A road oil fuel consumption variation, from the initial 20 million tons/year change to 16 million tons /year we can get $a=0, b=15, x_{0}=0, x=16$, according to the simple independent functions, as indicated by function 1, calculate the core problem degree of association:

$$
k_{0}\left(p_{02}\right)=k(16)=\frac{15-16}{15-0}=-0.0667>0
$$

i.e. with the strategy $S_{1}$ problem association degree $k\left(p_{02}\right)$ change to -0.0667 , as the association degree less than 0 , so the strategy is infeasible. Same logical we can get the following:

Core problem association degree by replace conversion $\varphi_{113}$ and conduction conversion (i.e. strategy $S_{2}$ )

$$
k_{0}\left(p_{03}\right)=k(12)=\frac{15-12}{15-0}=0.2>0
$$

Core problem association degree by replace conversion $\varphi_{113}$ and conduction conversion (i.e. strategy $S_{3}$ )

$$
k_{0}\left(p_{04}\right)=k(90)=\frac{15-80}{15-0}=-4.3333<0
$$

Core problem association degree by replace conversion $\varphi_{113}$ and conduction conversion (i.e. strategy $S_{4}$ ) 


$$
k_{0}\left(p_{05}\right)=k(11)=\frac{15-11}{15-0}=0.2667>0
$$

Core problem association degree by replace conversion $\varphi_{113}$ and conduction conversion (i.e. strategy $S_{5}$ )

$$
k_{0}\left(p_{06}\right)=k(17)=\frac{15-17}{15-0}=-0.1333<0
$$

Core problem association degree by replace conversion $\varphi_{113}$ and conduction conversion (i.e. strategy $S_{6}$ )

$$
k_{0}\left(p_{07}\right)=k(13)=\frac{15-13}{15-0}=0.1333>0
$$

Core problem association degree by replace conversion $\varphi_{113}$ and conduction conversion (i.e. strategy $S_{7}$ )

$$
k_{0}\left(p_{07}\right)=k(26)=\frac{15-26}{15-0}=-0.7333<0
$$

Core problem association degree by replace conversion $\varphi_{113}$ and conduction conversion (i.e. strategy $S_{8}$ )

$$
k_{0}\left(p_{07}\right)=k(18)=\frac{15-18}{15-0}=-0.2<0
$$

Through the calculation of relative strategy association degree, problem $S_{2}, S_{4}, S_{6}$ association degree is equal or greater than 0 , so the three strategies is feasible. Among them, strategy $S_{2}$ change the city A city center branches from 3 to 9; Strategy $S_{4}$ change city A main vehicle from bus to metro; Strategy $S_{6}$ change city A vehicle energy from gasoline to hybrid power.

\section{Strategy goodness evaluation}

Through calculation of strategy problem association degree, we can get the feasibility of the strategy. But different strategy has different goodness. Goodness measurement can be calculated by extension strategy goodness evaluation method. Here we do goodness evaluation on the three strategies: $S_{2}, S_{4}, S_{6}$

(1) Fix the measurement condition

Definite measurement condition set $M=\left\{M_{1}, M_{2}\right\}$, among them, characteristic element $M_{i}=\left(c_{i}, V_{i}\right), i=1,2$, and $V_{i}$ has been quantized. The measurement condition in this paper is $\mathrm{M} 1=($ fund input, V1), its value domain V1=[1,500]billion yuan, optimum value $x_{01}=1$ billion yuan; M2=(responding time, $\mathrm{V} 2$ ), and value domainV2=[1,20]year, optimum value $x_{02}=1$ year. And $M_{1}$ weight coefficient $\alpha_{1}=0.6$, $M_{2}$ weight coefficient $\alpha_{2}=0.4$, and $\sum_{i=1}^{2} \alpha_{i}=1$.

Among them strategy $S_{2}$ M12=(Fund input, 1000 billion yuan), M22=(responding time, 50 years).

Strategy $S_{4}$ M14=Fund input, 400 billion yuan), M24=(responding time, 50 years).

Strategy $S_{6}$ M16=Fund input, 600 billion yuan), M22=(responding time, 15 years).

(2) Goodness calculation

We now calculate relative strategy goodness through provided measurement condition and weight coefficient: First, calculate relative strategy goodness through function 1:

$$
K_{1}\left(S_{2}\right)=k(1000)=\frac{500-1000}{500-1}=-1.0020
$$




$$
\begin{gathered}
K_{2}\left(S_{2}\right)=k(50)=\frac{20-50}{20-1}=-1.5789 \\
K_{1}\left(S_{4}\right)=k(400)=\frac{500-400}{500-1}=0.2004 \\
K_{2}\left(S_{4}\right)=k(10)=\frac{20-10}{20-1}=0.5263 \\
K_{1}\left(S_{6}\right)=k(600)=\frac{500-600}{500-1}=-0.2004 \\
K_{2}\left(S_{6}\right)=k(15)=\frac{20-15}{20-1}=0.2632
\end{gathered}
$$

Second, calculate relative strategy standard conformity through function 2 .

$$
\max _{x \in V_{1}} K_{1}\left(S_{i}\right)=0.2004 \max _{x \notin V_{1}}\left|K_{1}\left(S_{i}\right)\right|=1.0020,
$$

among them $i=2,4,6$.

$$
\max _{x \in V_{2}} K_{2}\left(S_{i}\right)=0.5263 \max _{x \notin V_{2}}\left|K_{2}\left(S_{i}\right)\right|=1.5789,
$$

among them $i=2,4,6$.

$$
\begin{gathered}
k_{12}=k_{1}\left(S_{2}\right)=\frac{K_{1}\left(S_{2}\right)}{\max _{x \notin V_{1}}\left|K_{1}\left(S_{i}\right)\right|}=\frac{-1.0020}{1.0020}=-1 \\
k_{22}=k_{2}\left(S_{2}\right)=\frac{K_{2}\left(S_{2}\right)}{\max _{x \notin V_{2}}\left|K_{2}\left(S_{i}\right)\right|}=\frac{-1.5789}{1.5789}=-1 \\
k_{14}=k_{1}\left(S_{4}\right)=\frac{K_{1}\left(S_{4}\right)}{\max _{x \in V_{1}} K_{1}\left(S_{i}\right)}=\frac{0.2004}{0.2004}=1 \\
k_{24}=k_{2}\left(S_{4}\right)=\frac{K_{2}\left(S_{4}\right)}{\max _{x \in V_{2}} K_{2}\left(S_{i}\right)}=\frac{0.5263}{0.5263}=1 \\
k_{16}=k_{1}\left(S_{6}\right)=\frac{K_{1}\left(S_{6}\right)}{\max _{x \notin V_{1}}\left|K_{1}\left(S_{i}\right)\right|}=\frac{-0.2004}{1.0020}=-0.2 \\
k_{26}=k_{2}\left(S_{6}\right)=\frac{K_{2}\left(S_{6}\right)}{\max _{x \in V_{2}} K_{2}\left(S_{i}\right)}=\frac{0.2632}{0.5263}=0.5
\end{gathered}
$$

Last, calculation strategy goodness:

$$
\begin{gathered}
C_{S_{2}}=\sum_{i=1}^{2} k_{i 2} \alpha_{i}=-1 \quad C_{S_{4}}=\sum_{i=1}^{2} k_{i 4} \alpha_{i}=1 \\
C_{S_{6}}=\sum_{i=1}^{2} k_{i 6} \alpha_{i}=0.08
\end{gathered}
$$


We can get the conclusion that strategy $S_{2}$ goodness is -1, strategy $S_{4}$ goodness is 1, strategy $S_{6}$ goodness is 0.08 , and we can see that $S_{4}$ goodness is the best one among the three strategies. Strategy $S_{2}$ goodness less than 0 , although strategy feasible, but not advisable. So we use extension strategy generating method to solve the incompatible problem of excessive road oil fuel consumption by changing the city A main vehicle from bus to metro and realize city A road oil fuel consumption from 20 million tons/year to 11 million tons/year.

\section{Conclusion}

Extension strategy generating method is a kind of strategy generating method based on extenics and imitate human thinking model to solve incompatible problem. This paper introduced this method on the research of excessive road oil fuel consumption problem and realized the strategy generating process by formalization \& quantification. Thereby, it provides reference to the reduction of the road oil fuel consumption \& the set up sustainable development of city transportation.

\section{References}

[1] Weihua Zhang, Hui Wang, Gang Hu. City development strategy based on low transportation energy consumption. Beijing: Road traffic technology. 2003.(in chinese)

[2] Lixi Li, Chunyan, Yang, Huawen Li. Extension strategy generating systerm [M] (The First Edition).Beijing: Science Press, 2006.(in chinese).

[3] Chunyan Yang, Wen Cai. Extension engineering [M] (The First Edition).Beijing: Science Press, 2007.(in chinese).

[4] Wen Cai. Chunyan Yang, Extension engineering method [M] (The First Edition).Beijing: Science Press, 1997.(in chinese)

[5] Wen Cai. Chunyan Yang, Bin He, Extension logic rudiment [M] Science Press, 2003.(in chinese)

[6] Guangzi Ye, Weihua Li. Xihua Zhang. Extension strategy generating system study and implement on air pollution control. Guangdong: Guang: Journal of Guangdong University of Technology, 2007.(in chinese)

[7] Qitang Wu. Develop small-displacement \& multi-energy vehicles-----Study new < Automobile industry development policy> 7th, Shanghai: Cars, Parts $\&$ Vehicles, 2004.(in chinese). 